\title{
Defect Automatic Identification of Eddy Current Pulsed Thermography
}

\author{
Kai Chen, Libing Bai, Yifan Chen, Yuhua Cheng, Shulin Tian, and Peipei Zhu \\ School of Automation Engineering, University of Electronic Science and Technology of China, 2006 Xiyuan Avenue, \\ Chengdu, Sichuan 611731, China
}

Correspondence should be addressed to Libing Bai; libing.bai@uestc.edu.cn

Received 18 July 2014; Revised 31 October 2014; Accepted 31 October 2014; Published 31 December 2014

Academic Editor: Ignacio R. Matias

Copyright (C) 2014 Kai Chen et al. This is an open access article distributed under the Creative Commons Attribution License, which permits unrestricted use, distribution, and reproduction in any medium, provided the original work is properly cited.

Eddy current pulsed thermography (ECPT) is an effective nondestructive testing and evaluation (NDT\&E) technique, and has been applied for a wide range of conductive materials. Manual selected frames have been used for defects detection and quantification. Defects are indicated by high/low temperature in the frames. However, the variation of surface emissivity sometimes introduces illusory temperature inhomogeneity and results in false alarm. To improve the probability of detection, this paper proposes a twoheat balance states-based method which can restrain the influence of the emissivity. In addition, the independent component analysis (ICA) is also applied to automatically identify defect patterns and quantify the defects. An experiment was carried out to validate the proposed methods.

\section{Introduction}

A review of current literature shows that thermography is applicable to a wide range of materials [1-6]. Eddy current pulsed thermography (ECPT) has been attempted in previous studies. The temperature distribution around a crack with different penetration depths in metallic materials was investigated in [7]. The potential for small defects detection in components of complex geometry such as compressor blades and low pressure turbine vanes was discussed in [8]. The probability of detection (POD) of fatigue cracks in steel, titanium, and nickel-based superalloy was estimated in [9]. Multiple cracks from rolling contact fatigue in rail track in a single measurement were detected in [10]. In all of these works, defects were detected and quantified using manually selected frames. Defects were indicated by high/low temperature spot in the frames. In the in situ application, the units under test always have oil or the oxidation layer on the surface, which changes the thermal emissivity significantly. The variation of surface emissivity sometimes introduces illusory temperature inhomogeneity and results in false alarm.

To remove the influence of surface emissivity variation, several methods have been attempted in previous studies. Noethen et al. put a very thin water film on sample surface to homogenize the emissivity of the oxidized surface [11]. Avdelidis and Almond sprayed black paint on aluminium sample to eliminate high reflectance and raise emissivity [12]. These methods can improve the homogeneity of the surface emissivity. And to a certain extent, the signal-to-noise ratio of the image is improved. However, water or black paint not only pollutes the sample, but also increases the cost and complexity of the test procedure.

Shepard proposed thermographic signal reconstruction (TSR) based flash thermography to reduce the influence of the thermal emissivity [13]. Maldague et al. inhibited the thermal emissivity variation influence by the pulse phase infrared thermography method $[14,15]$. Chatterjee and Tuli removed influence of nonuniform heating and surface emissivity variation using a Fourier transformation based image reconstruction algorithm [16]. Fourier transformation attracts a wide application in flash stimulation thermal imaging [17], lockin thermography [16]. However, the defect information is separated into several frequency components, which conceals the defect quantification information conceived in transient response [18-20].

To solve the problem mentioned above, this paper combines the authors' two previous proposed methods: two-heat balance states [21] and blind source separation [22], which 
promises defect automatic identification in the in situ application. The rest of the paper is organized as follows: Section 2 discusses the implementation of emissivity inhibition and automatic detection; Section 3 introduces the experimental setup; Sections 4 and 5 present the experiment results and the conclusions.

\section{Theoretical Considerations}

2.1. The Theory of the Two-Heat Balance States-Based Method. The equation of the theory of the two-heat balance statesbased method can be described as

$$
j_{i, j}(t)=\frac{j_{i, j}^{*}(t)}{j_{i, j}^{*}\left(t_{1}\right)-j_{i, j}^{*}\left(t_{0}\right)}=\frac{T_{i, j}^{4}(t)}{T_{1}^{4}-T_{0}^{4}},
$$

where $t$ is time, $T_{i, j}(t)$ denotes the temperature response of the area corresponding to pixel $(i, j), T_{0}$ equals the ambient temperature, and $T_{1}$ equals the temperature of equilibrium state. $j_{i, j}(t)$ is independent of the emissivity and describes the temperature changing process [21].

2.2. Quantifying Independence Using Kurtosis. As there is only one infrared camera in ECPT, this refers to a typical single channel source separation problem. The mathematical model can be described as

$$
\mathbf{Y}(t)=\sum_{i=1}^{N_{s}} m_{i} \mathbf{X}_{i}(t)
$$

The ICA learning algorithm which is equivalent to searching for the linear transformation that makes the components as statistically independent as possible has been used to separate the thermal response signal [23-25]. The sources can be estimated as

$$
\widehat{\mathbf{X}}_{\mathrm{ICA}}^{\prime}(t)=\widehat{\mathbf{W}}_{\mathrm{ICA}} \mathbf{U}_{T \times N_{s}}^{T},
$$

where $\widehat{\mathbf{W}}_{\text {ICA }}$ is the linear transformation matrix [22]. The mixing model can be shown in Figure 1.

In the independent components, the area without defects is much bigger than the other area and contains amount of pixels, which shows more centralized in statistics. For a Gaussian random variable, the kurtosis is zero. For most of other cases, kurtosis is nonzero for non-Gaussian random variables. The bigger the kurtosis is, the more abundant the information of defect is. Therefore the kurtosis can be applied to extract the defects automatically. The kurtosis of thermal images can be described as

$$
\operatorname{kurt}\left(X_{i}(t)\right)=\frac{\sum_{j=1}^{M} \sum_{k=1}^{N}\left[X_{i, j k}(t)-\bar{X}_{i}(t)\right]^{4}}{(M N-1) \sigma^{4}}-3,
$$

where $\bar{X}_{i}(t)$ is the mean of the thermal image $X_{i}(t), \sigma$ is the standard deviation of $X_{i}(t)$, and $M$ and $N$ are the pixel of thermography.

The process of automatic detection can be summarized as shown in Figure 2.

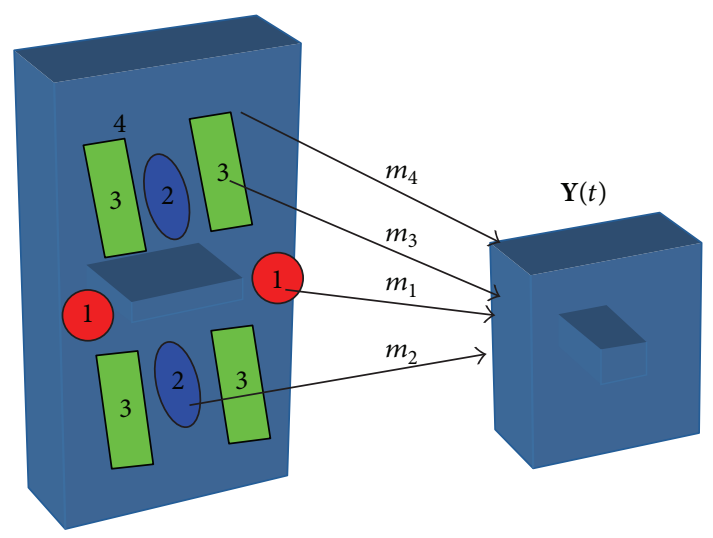

FIGURE 1: The mixing model of ICA.

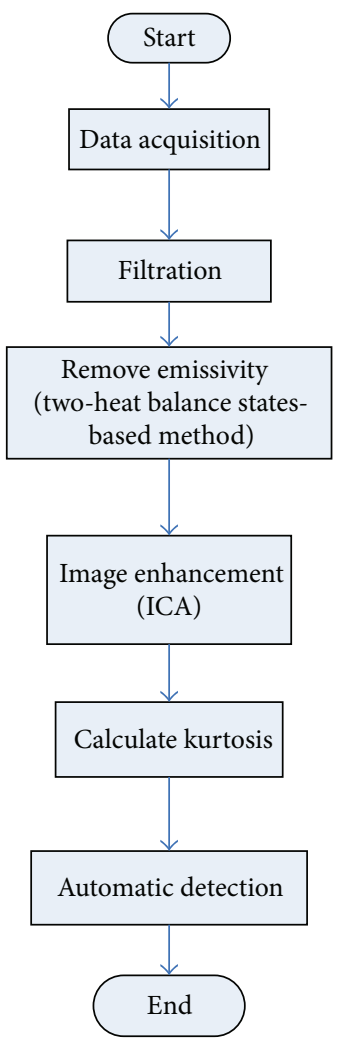

Figure 2: Flow chart of automatic detection.

\section{Experiment Setup and Sample}

The experimental setup is shown in Figure 3. The induction heater used for coil excitation has a maximum excitation power of $15 \mathrm{~kW}$, a maximum current of $700 \mathrm{Arms}$, and an excitation frequency range of $30-100 \mathrm{kHz}(150 \mathrm{Arms}$ and $80 \mathrm{kHz}$ are used in this study). The system has a quoted rise time (from the start of the heating period to full power) of $250 \mathrm{~ms}$, which was verified experimentally. Water cooling of coil is implemented to counteract direct heating of the coil.

The IR camera, FLIR A655sc, is an uncooled camera with $480 \times 640$ array. This camera has a sensitivity of $<50 \mathrm{mK}$ and a maximum full frame rate of $50 \mathrm{~Hz}$, with the option to increase 


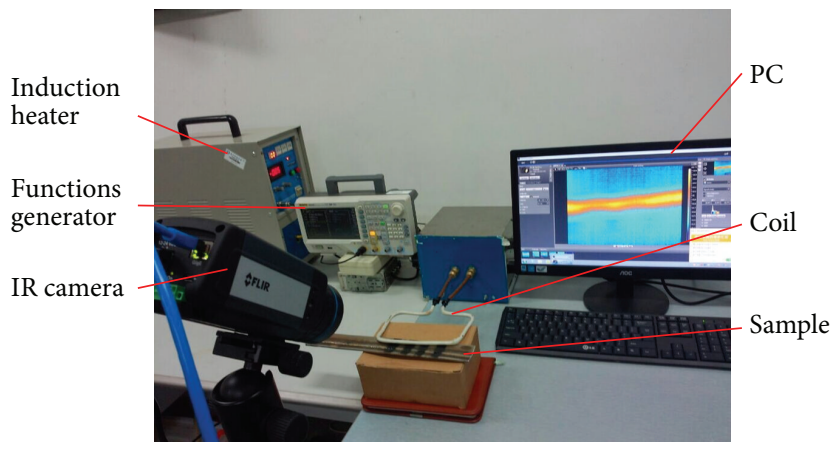

FIGURE 3: Experiment setup.

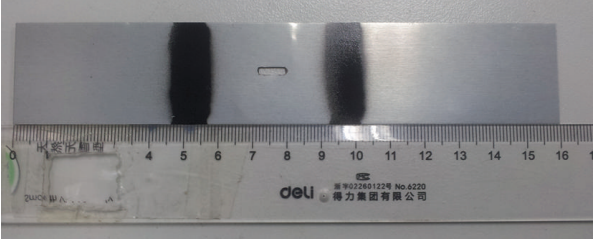

(a)

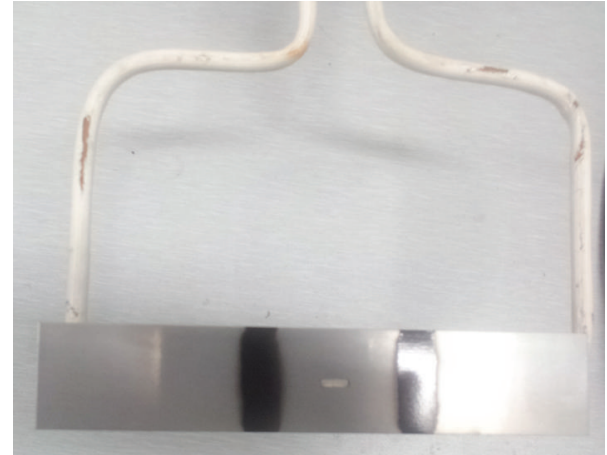

(b)

Figure 4: (a) Steel sample with slot. (b) Directional excitation coil.

frame rate with windowing of the image. A rectangular coil is constructed to apply directional excitation. This coil is made of inner diameter $7 \mathrm{~mm}$ high conductivity hollow copper tube. In the experiment, only one edge of the rectangular coil is used to stimulate eddy current to the underneath sample. In this study, the frame rate is $100 \mathrm{~Hz}$ with $240 \times 640$ array, and $2.6 \mathrm{~s}$ videos are recorded in the experiments.

A silicon steel sheet sample $(0.33 \mathrm{~mm} \times 30 \mathrm{~mm} \times 160 \mathrm{~mm})$ with a slot of $8 \mathrm{~mm}$ length and $2 \mathrm{~mm}$ width is prepared (Figure 4(a)). There are equally spaced shinning and black stripes on the sample surface. The shinning strips are the polished area and the black strips are the area sprayed black painting. A $300 \mathrm{~ms}$ heating duration is selected for inspection, which is long enough to elicit an observable heat pattern. The cooling time is $2.2 \mathrm{~s}$, which is long enough to ensure the sample reaching a new thermal equilibrium state. The excitation coil is placed at the back of the sample and parallels the sample, as shown in Figure 4(b).

\section{Results and Discussion}

4.1. The Removement of the Emissivity. By setting $t_{1}=2.5 \mathrm{~s}$, the transient response is corrected using (1). The results are shown in Figure 5. Figures 5(a) and 5(b) show the original temperature distribution and transient responses. There is a great difference between Pos1 and Pos2. Figure 5(c) shows the corrected temperature distribution at the end of heating
(0.3 s). The black stripes are almost invisible. Pos1 and Pos2 show nearly similar temperature. The transient responses of the 3 positions are shown in Figure 5(d). Pos3 has a high rising rate in the heating phase and high falling rate at the cooling phase; Pos1 has low rising rate in the heating phase and low falling rate in the cooling phase; Pos 2 almost coincided with Posl. These are in line with the transient response acquired from homogeneity surface [22].

4.2. Automatic Defect Independent Component (IC) Identification Using Kurtosis. In order to realize the automatic detection of the defect, the images which have been processed by two-heat balance states-based method are processed by ICA. Table 1 shows the four ICs and the corresponding estimated mixing vectors. It is noted that IC2, IC3, and IC4 highlight different parts of the excitation coil and several relative areas due to reflection of the shining sample surface. In addition, IC1 highlights the area including slot tips and its estimated mixing vector shows that the high rising and falling rate in heating and cooling phase around the slot tip are in line with the previous analysis [22]. The kurtosis is also calculated using (4). The kurtosis of IC1 is the maxim value (25.7) over other ICs (IC2 1.8; IC3 5.6; IC4 3.8) that directly indicates the defect area. Hence, the kurtosis can be calculated to detect the defects automatically.

To further verify the above method, one subsurface defect and three surface defects in stainless steel welds are employed 
TABLE 1: Independent components and estimated mixing vectors.

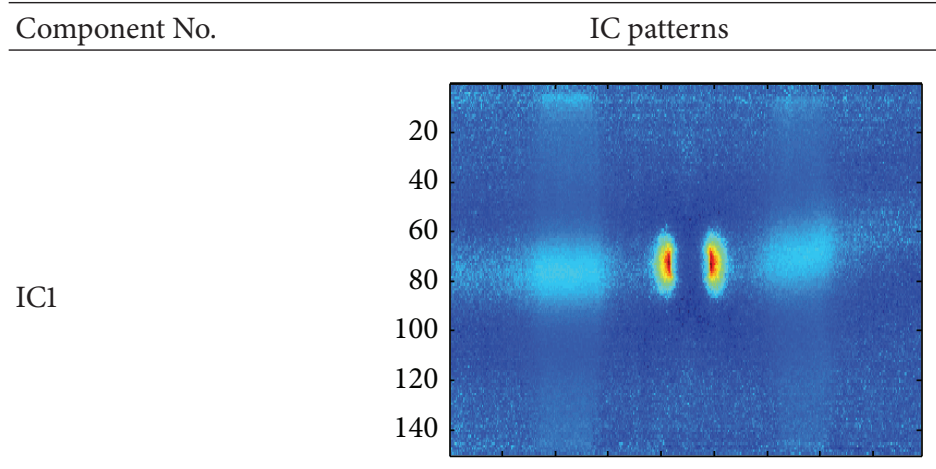

50100150200250300350400

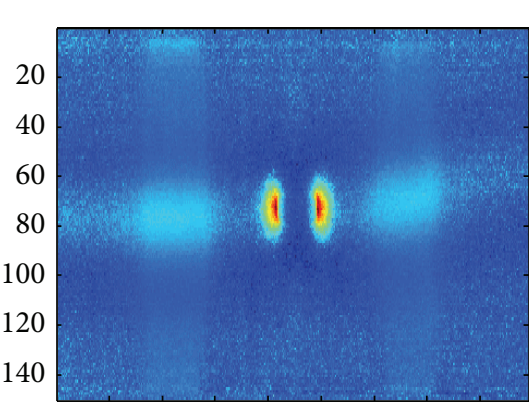

50100150200250300350400

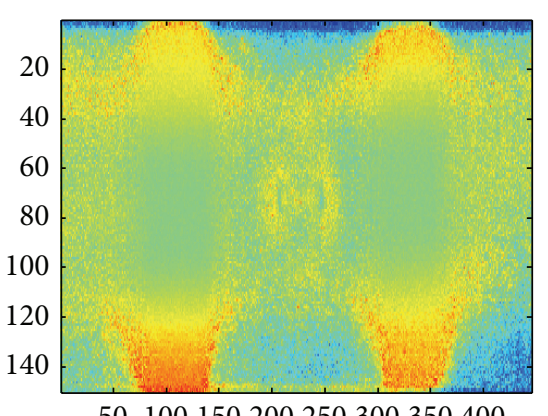

50100150200250300350400

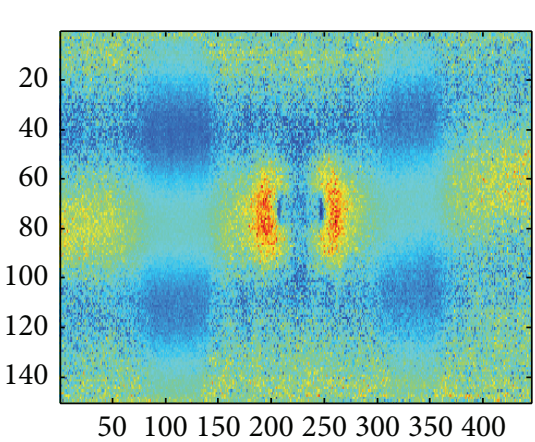

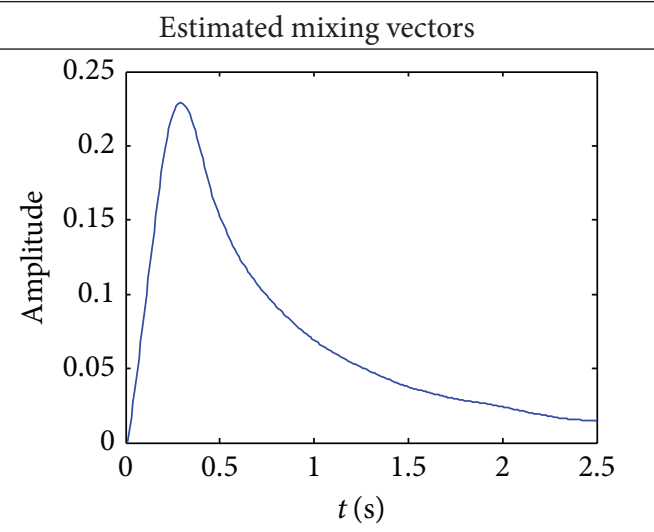
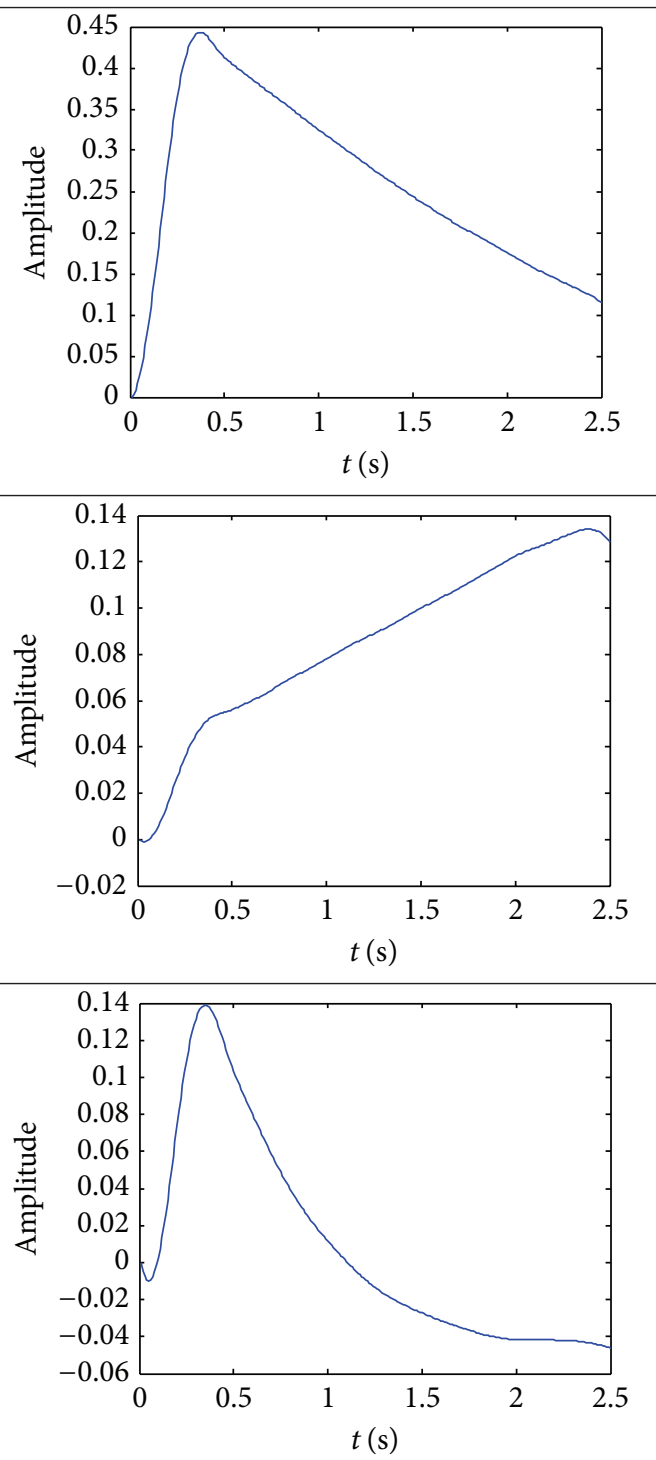


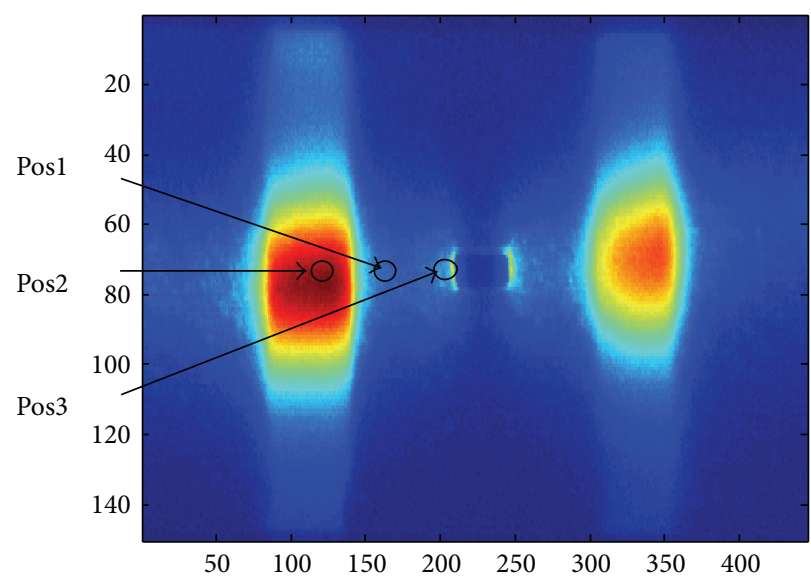

(a)

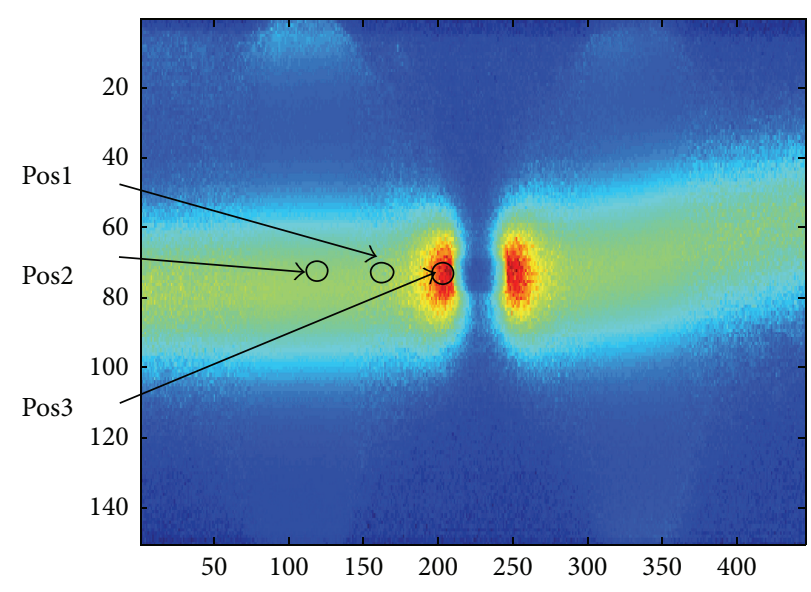

(c)

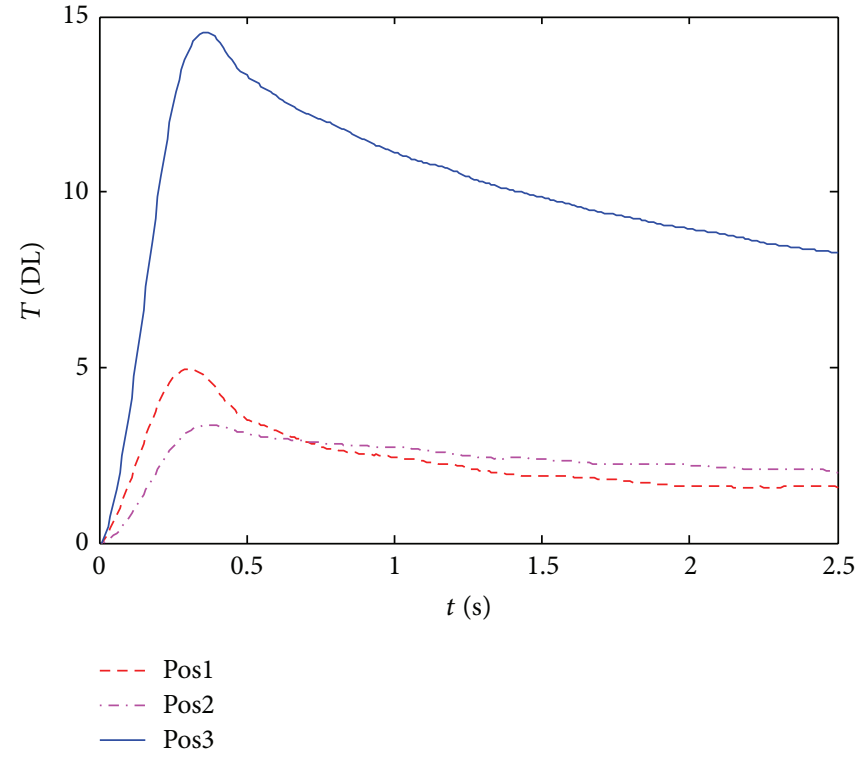

(b)

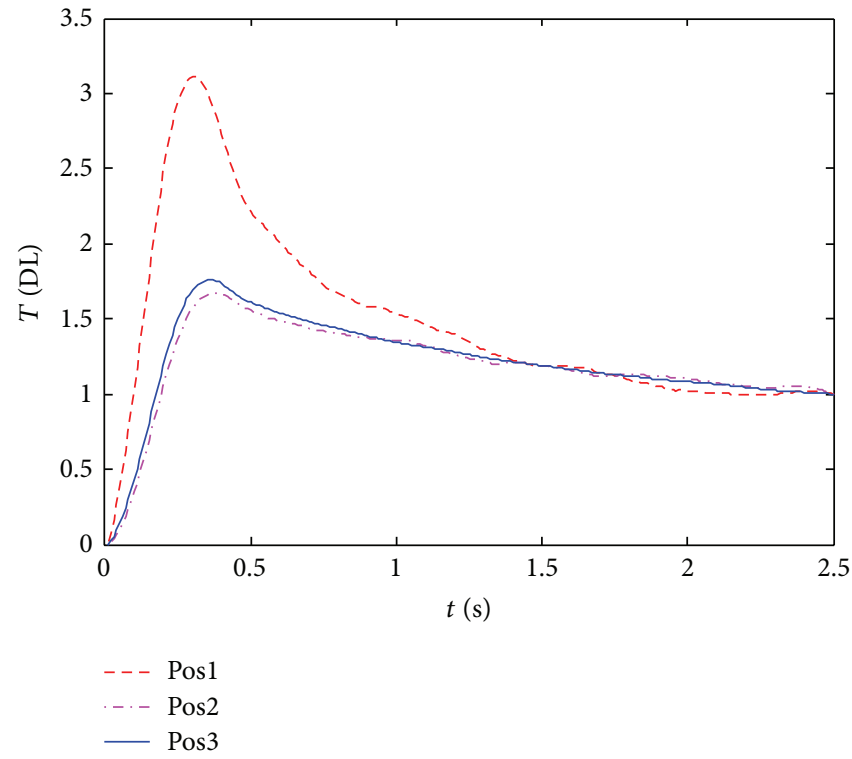

(d)

FIGURE 5: (a) Original image, (b) original transient responses, (c) corrected infrared image at $0.3 \mathrm{~s}$, and (d) processed transient responses of different positions.

for testing. All testing was carried out from the top side. The results are shown in Table 2. There is a slot of $5 \mathrm{~mm}$ length and $1 \mathrm{~mm}$ width in sample (1). The IC1 highlights the area including defect, and its kurtosis is the maxim value (67.9) over other ICs (IC2 12.7; IC3 6.1; IC4 8.8). There is a subsurface defect of $5 \mathrm{~mm}$ length, $1 \mathrm{~mm}$ width, and $2 \mathrm{~mm}$ depth in sample (2). The IC1 highlights the area including defect, and its kurtosis is the maxim value (40.0) over other ICs (IC2 11.4; IC3 11.3; IC4 3.6). There are two surface defects of $1 \mathrm{~mm}$ diameter, $3 \mathrm{~mm}$ and $5 \mathrm{~mm}$ depth in sample (3). Similarly, the IC1 highlights the area including defects, and its kurtosis is the maxim value (62.8) over other ICs (IC2 16.1;
IC3 6.2; IC4 3.3). It is clear that the proposed method can detect the defects effectively and automatically.

\section{Conclusion}

In this paper, two-heat balance states-based method and blind source separation are combined for defect automatic identification. A validated experiment is carried out. The conclusions can be drawn as follows.

(1) The temperature and emissivity are separated by the two-heat balance states-based method. 
TABLE 2: Samples and independent component.

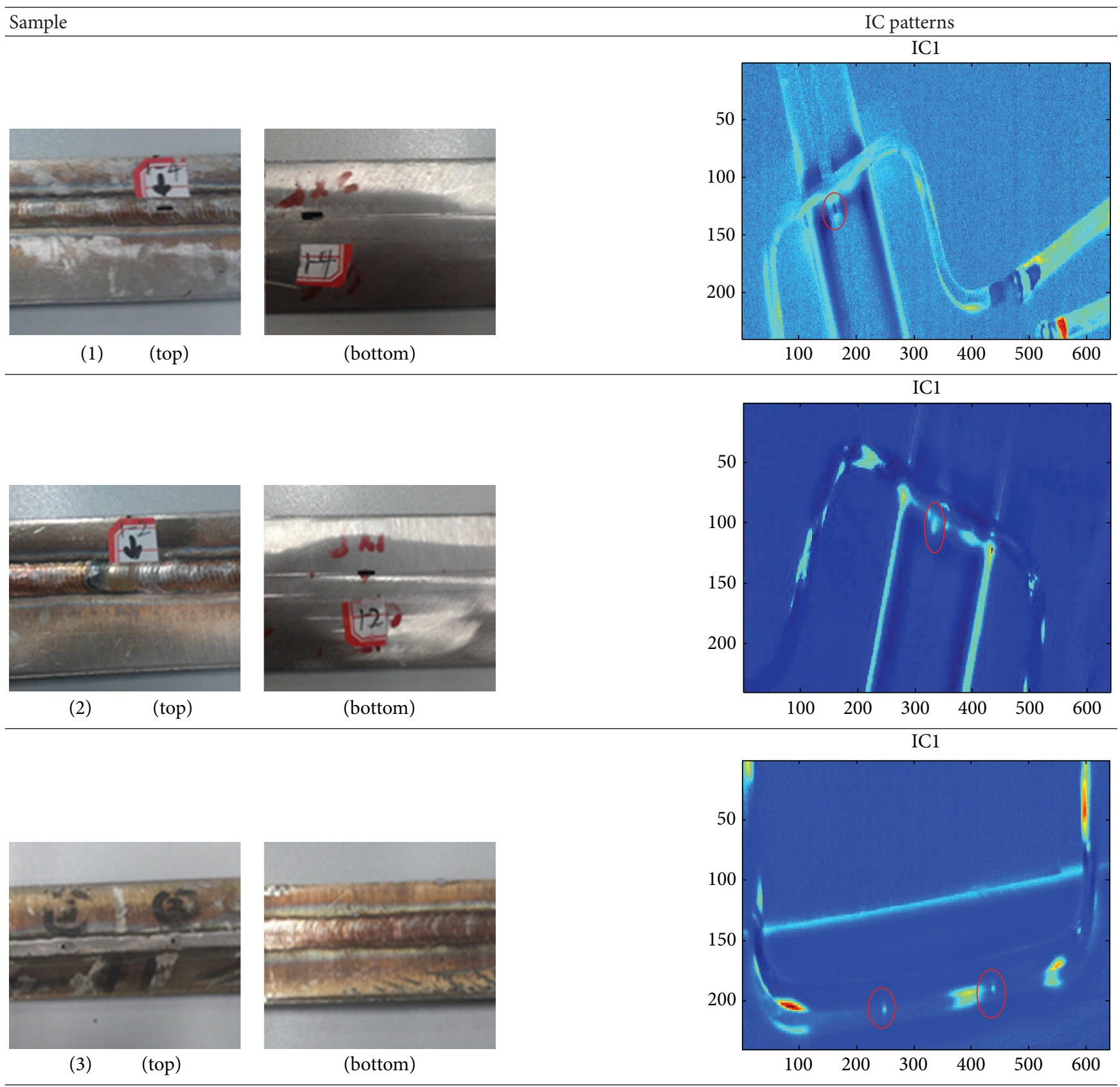

(2) The IC highlighting the defect can also be identified using kurtosis. The singular pattern around crack tips has a high temperature in small area and high temperature gradient around the edge. This results in extremely super-Gaussian and maximum kurtosis value.

\section{Conflict of Interests}

The authors declare that there is no conflict of interests regarding the publication of this paper.

\section{Acknowledgments}

This paper is supported by "the Fundamental Research Funds for the Central Universities" (ZYGX2012YB029) and "the National Natural Science Foundation of China" (Grant nos. 61102141 and 51407024).

\section{References}

[1] N. P. Avdelidis, B. C. Hawtin, and D. P. Almond, "Transient thermography in the assessment of defects of aircraft composites," NDT and E International, vol. 36, no. 6, pp. 433-439, 2003. 
[2] Z. Zeng, N. Tao, L. Feng, and C. Zhang, "Specified value based defect depth prediction using pulsed thermography," Journal of Applied Physics, vol. 112, no. 2, Article ID 023112, 2012.

[3] C. Meola, "Nondestructive evaluation of materials with rear heating lock-in thermography," IEEE Sensors Journal, vol. 7, no. 10, pp. 1388-1389, 2007.

[4] S. Huth, O. Breitenstein, A. Huber, and U. Lambert, "Localization of gate oxide integrity defects in silicon metal-oxidesemiconductor structures with lock-in IR thermography," Journal of Applied Physics, vol. 88, no. 7, pp. 4000-4003, 2000.

[5] V. S. Ghali, N. Jonnalagadda, and R. Mulaveesala, “Threedimensional pulse compression for infrared nondestructive testing," IEEE Sensors Journal, vol. 9, no. 7, pp. 832-833, 2009.

[6] N. Biju, N. Ganesan, C. V. Krishnamurthy, and K. Balasubramaniam, "Simultaneous estimation of electrical and thermal properties of isotropic material from the tone-burst eddy current thermography (TBET) time-temperature data," IEEE Transactions on Magnetics, vol. 47, no. 9, pp. 2213-2219, 2011.

[7] B. Oswald-Tranta and G. Wally, "Thermo-inductive surface crack detection in metallic materials," in Proceedings of the 9th European Conference on Non-Destructive Testing (ECNDT '06), paper We.3.8.3, Berlin, Germany, September 2006.

[8] G. Zenzinger, J. Bamberg, W. Satzger, and V. Carl, “Thermographic crack detection by eddy current excitation," Nondestructive Testing and Evaluation, vol. 22, no. 2-3, pp. 101-111, 2007.

[9] B. Weekes, D. P. Almond, P. Cawley, and T. Barden, "Eddycurrent induced thermography-probability of detection study of small fatigue cracks in steel, titanium and nickel-based superalloy," NDT and E International, vol. 49, pp. 47-56, 2012.

[10] J. Wilson, G. Y. Tian, I. Mukriz, and D. Almond, "PEC thermography for imaging multiple cracks from rolling contact fatigue," NDT \& E International, vol. 44, no. 6, pp. 505-512, 2011.

[11] M. Noethen, K.-J. Wolter, and N. Meyendorf, "Surface crack detection in ferritic and austenitic steel components using inductive heated thermography," in Proceedings of the 33rd International Spring Seminar on Electronics Technology: Polymer Electronics and Nanotechnologies: Towards System Integration (ISSE '10), pp. 249-254, Warsaw, Poland, May 2010.

[12] N. P. Avdelidis and D. P. Almond, "Transient thermography as a through skin imaging technique for aircraft assembly: modelling and experimental results," Infrared Physics and Technology, vol. 45, no. 2, pp. 103-114, 2004.

[13] S. M. Shepard, "Flash thermography of aerospace composites," in Proceedings of the 4th Pan American Conference for NDT, Buenos Aires, Argentina, October 2007.

[14] X. Maldague and S. Marinetti, "Pulse phase infrared thermography," Journal of Applied Physics, vol. 79, no. 5, pp. 2694-2698, 1996.

[15] Y. He, G. Tian, M. Pan, and D. Chen, "Eddy current pulsed phase thermography and feature extraction," Applied Physics Letters, vol. 103, no. 8, Article ID 084104, 2013.

[16] K. Chatterjee and S. Tuli, "Image enhancement in transient lock-in thermography through time series reconstruction and spatial slope correction," IEEE Transactions on Instrumentation and Measurement, vol. 61, no. 4, pp. 1079-1089, 2012.

[17] J.-C. Krapez, L. Spagnolo, M. Frieß, H.-P. Maier, and G. Ü. Neuer, "Measurement of in-plane diffusivity in nonhomogeneous slabs by applying flash thermography," International Journal of Thermal Sciences, vol. 43, no. 10, pp. 967-977, 2004.
[18] Y. He, M. Pan, and F. Luo, "Defect characterisation based on heat diffusion using induction thermography testing," The Review of Scientific Instruments, vol. 83, no. 10, Article ID 104702, 2012.

[19] L. Bai and G. Y. Tian, "Stress measurement using pulsed eddy current thermography," in Proceedings of the 51st Annual Conference of the British Institute of Non-Destructive Testing (BINDT '12), Daventry, UK, 2012.

[20] L. Cheng and G. Y. Tian, "Surface crack detection for carbon fiber reinforced plastic (CFRP) materials using pulsed eddy current thermography," IEEE Sensors Journal, vol. 11, no. 12, pp. 3261-3268, 2011.

[21] L. Bai, S. Tian, Y. Cheng, G. Y. Tian, Y. Chen, and K. Chen, "Reducing the effect of surface emissivity variation in eddy current pulsed thermography," IEEE Sensors Journal, vol. 14, no. 4, pp. 1137-1142, 2014.

[22] L. Bai, B. Gao, G. Y. Tian, W. L. Woo, and Y. Cheng, "Spatial and time patterns extraction of eddy current pulsed thermography using blind source separation," IEEE Sensors Journal, vol. 13, no. 6, pp. 2094-2101, 2013.

[23] M. Pan, Y. He, G. Tian, D. Chen, and F. Luo, "Defect characterisation using pulsed eddy current thermography under transmission mode and NDT applications," NDT and E International, vol. 52, pp. 28-36, 2012.

[24] L. Cheng, B. Gao, G. Y. Tian, W. L. Woo, and G. Berthiau, "Impact damage detection and identification using eddy current pulsed thermography through integration of PCA and ICA," IEEE Sensors Journal, vol. 14, no. 5, pp. 1655-1663, 2014.

[25] Y. He, G. Tian, M. Pan, and D. Chen, "Impact evaluation in carbon fiber reinforced plastic (CFRP) laminates using eddy current pulsed thermography," Composite Structures, vol. 109, no. 1, pp. 1-7, 2014. 

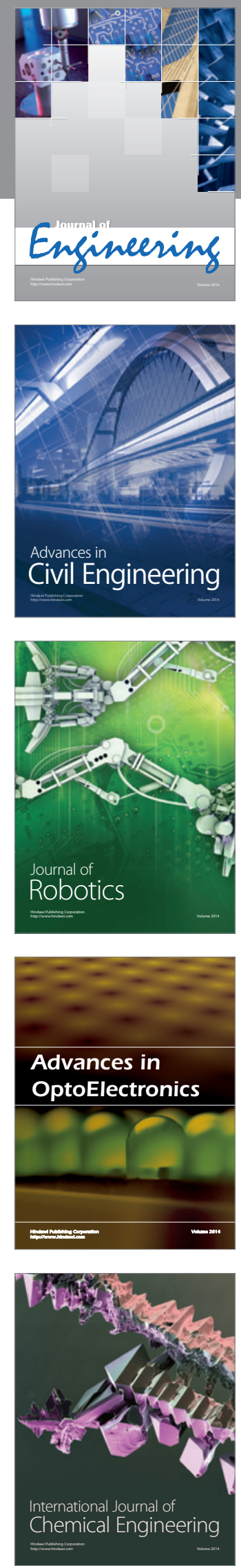

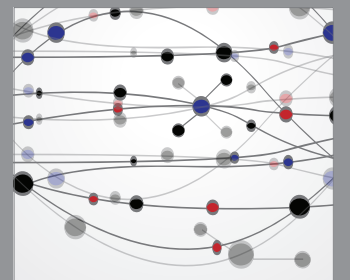

The Scientific World Journal
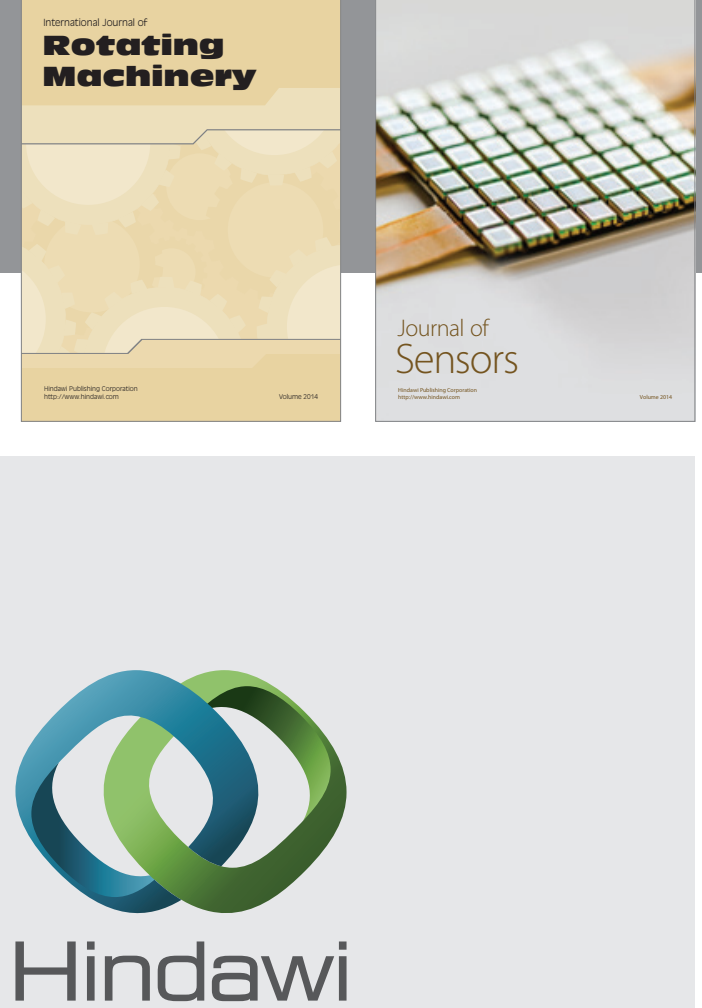

Submit your manuscripts at http://www.hindawi.com
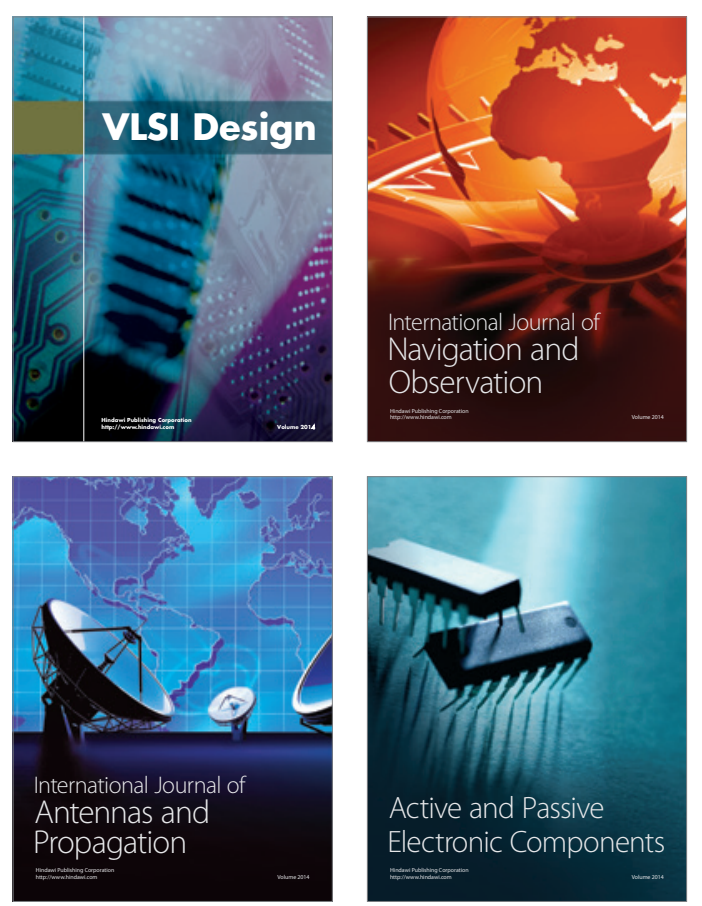
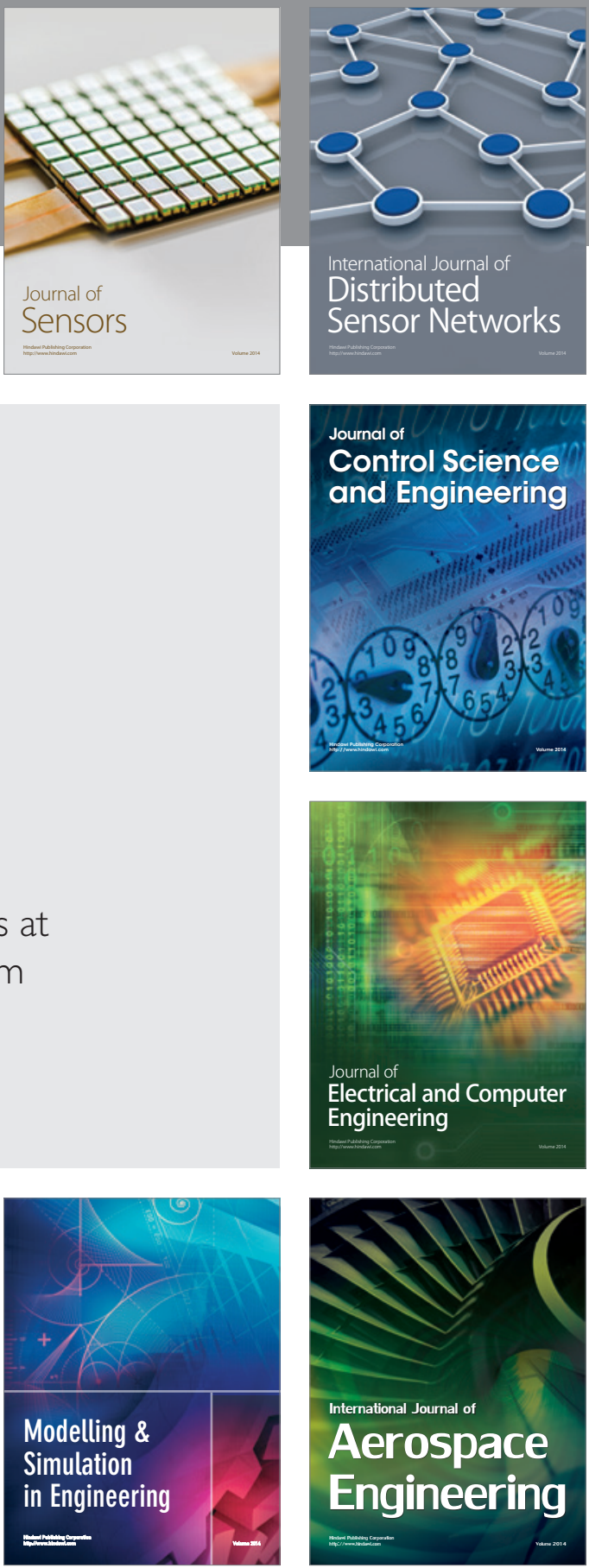

Journal of

Control Science

and Engineering
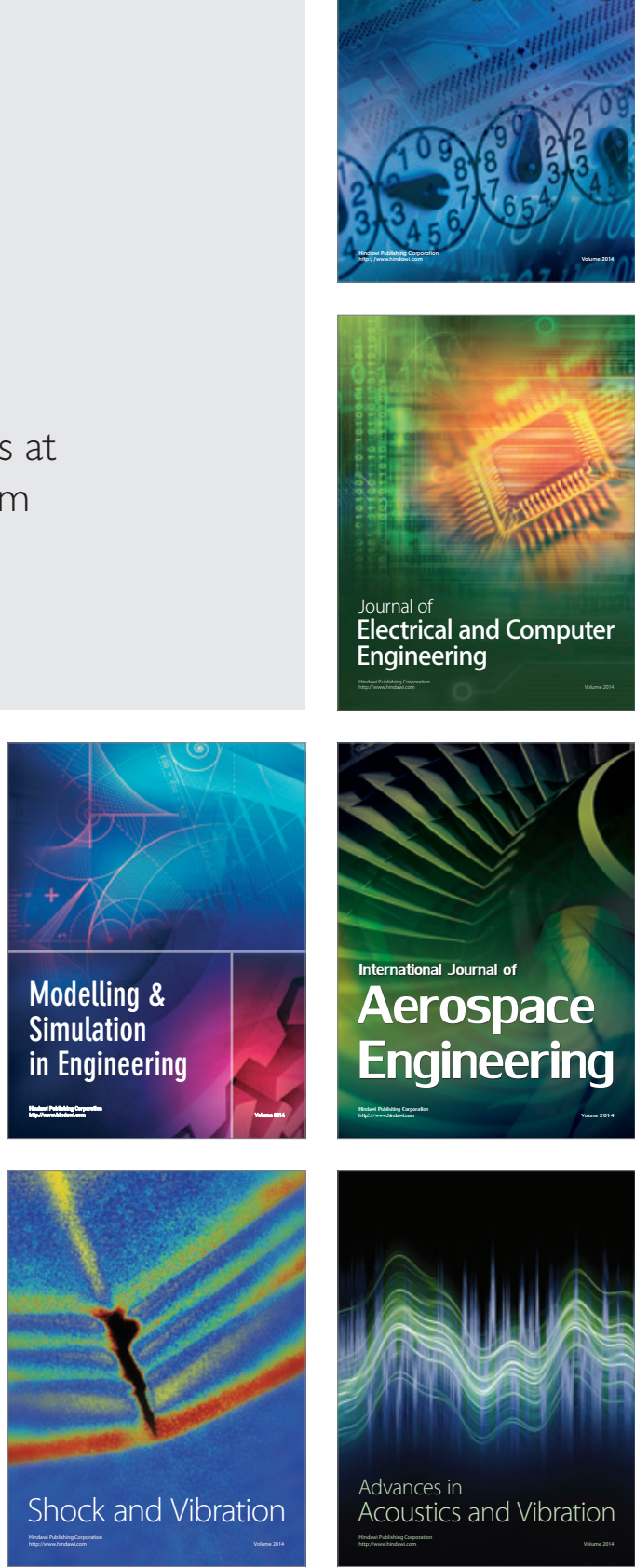